\title{
The Perturbation Toxic Effect of Graphene on HepG2 Cell
}

\author{
Guozheng Jiao ${ }^{1 \mathrm{a},}$, Xin $\mathrm{Li}^{\mathrm{a}, \mathrm{b}^{*}}$, Junqiang Qiu ${ }^{3 \mathrm{c}}$, Ning Zhang ${ }^{4 \mathrm{c}}$, Hongying $\mathrm{Xu}^{5 \mathrm{c}}$ and \\ Shumin $\mathrm{Liu}^{6, \mathrm{C}^{*}}$ \\ ${ }^{1}$ Department of Chemistry, Harbin Institute of Technology, Harbin 150090, China \\ ${ }^{2}$ State Key Lab of Urban Water Resource and Environment, Harbin Institute of Technology, Harbin \\ 150090, \\ ${ }^{3}$ nstitute of Traditional Chinese Medicine, Key Laboratory of Chinese Materia Medica, Ministry of \\ Education, Heilongjiang University of Chinese Medicine, Harbin 150040, China \\ a E-mail:lixin@hit.edu.cn, ${ }^{c}$ E-mail:keji-liu@163.com, \\ * the corresponding author
}

Keywords: graphene, HepG2, metabolomics, toxicity

Abstract. How to accurately and comprehensively unveil the toxic effect of graphene on the cells have always received extensive attention by many researchers. Owing to the opposition results in classical assay approaches, some researchers have attempted to adopt advanced analysis techniques. Our group successful performed mass spectrometry-based metabolomics to investigate the toxic effect of graphene on the HepG2. In this analysis platform, by pattern recognition and metabolism analysis, we acquired twelve potential biomarkers and two main disturbed metabolism pathway involving in glutathione metabolism and arginine and proline metabolism. Our findings demonstrated that the graphene stimulate the HepG2 cell and results in breaking the homeostasis and perturbation the metabolism pathway, we should focus on this disturbance from the graphene.

\section{Introduction}

Graphene, and its derivatives, owing to having the unique chemical and physical property, are wieldy studied, manufactured, as well as used, not only in photoelectricity physical field, but also in biomedical scope. So the chance of exposure of it for the commonly people are more and more pronounced, the potential health risk and hazard are focused by people. Although the classical in vitro toxic assay methods are popular in testing the purity compounds in biomedical, many researchers still have found that some assay methods in vitro are disturbed by various modes in testing nanomaterials, such as, disturbance the assay agentias including adsorption or/and stimulus redox, interference in detection optical route, invoking cells inactive sensitivity assay agentias and so on. The graphene nanomaterials have also been found disturbing some assay approaches by reported similar pattern. Therefore, in vitro assay too many results were revealed the inconsonant and irrelevant and aroused too much controversy about the toxic or no-toxic of the graphene nanomaterials.[1] Some researchers have attempted to adopt advanced analysis techniques to assay the toxic of graphene nanomaterials. Previously, we have successfully performed the metabolomics to access the toxic effect of water soluble graphene on the HepG2 cell.[2] Hence, once again we carried out the metabolomics platform to investigate the toxicity of graphene on HepG2.

In the current study, we adopted the UPLC-Q-TOF-MS to assay the metabolites of HepG2 cells treated with graphene, then combined the pattern recognition with the biological events to detailed expound the disturbance metabolites and perturbation metabolism pathway. We acquired twelve metabolites and two main pathway disturbed by graphene including glutathione metabolism and arginine and proline metabolism. It demonstrated that the graphene have some extent disturbance for the HepG2 cell, in vivo usage, some great attention should been taken. 


\section{Results and Discussion}

\section{Pattern recognition}

a)

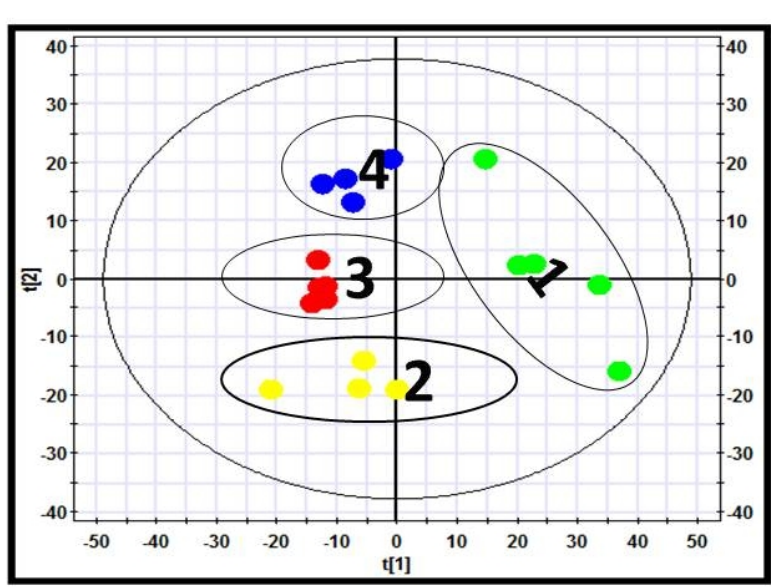

c)

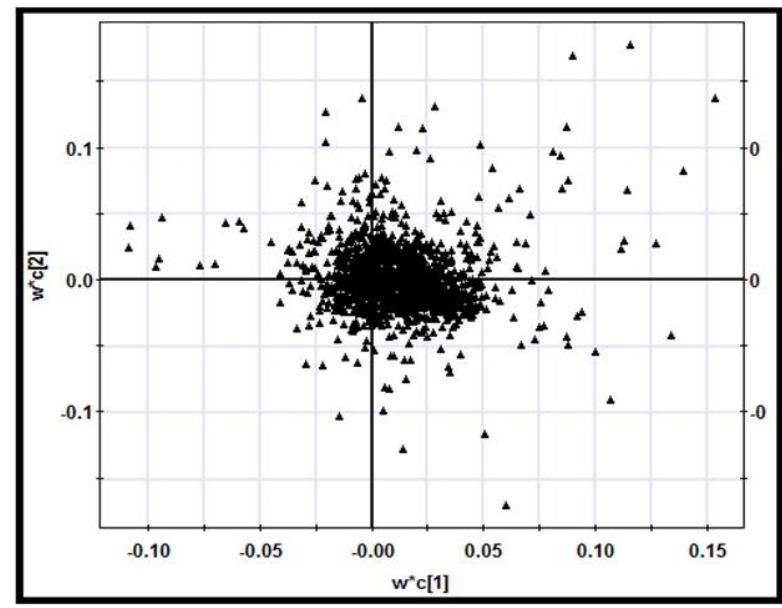

b)

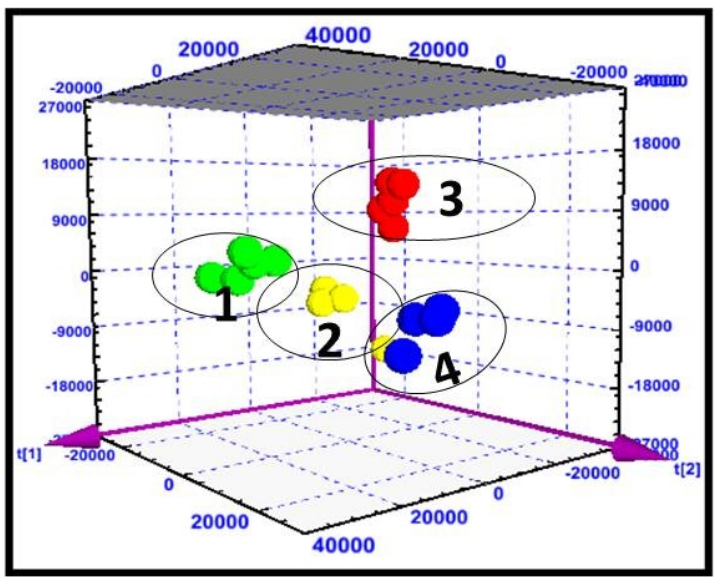

d)

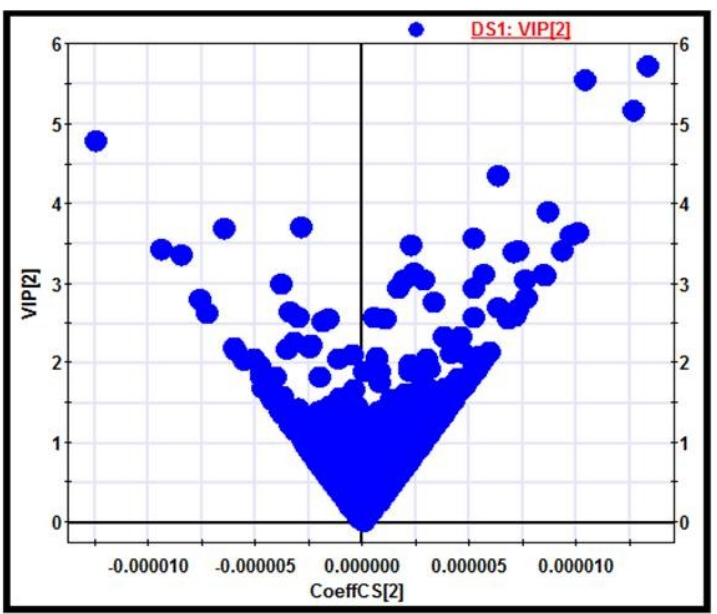

Fig. 1 The metabolic profile of Chang liver cell treated with graphene. $a$ ). the score plot of PCA; $b$ ). 3D-score plot of PLSD A; $c$ ). Loading plot of PLSD_A; $d$ ). The VIP score plot of PLSD_A. circle1: control dose group (PBS); circle2: low dose group (graphene, 5 $\mu \mathrm{g} \cdot \mathrm{mL}^{-1}$ ); circle3: middle dose group (graphene, $40 \mu \mathrm{g} \cdot \mathrm{mL}^{-1}$ ); circle4: high dose group (graphene, $1.00 \mu \mathrm{g} \cdot \mathrm{mL}^{-1}$ ).

All the mass data were input the masslynx 4.1(Waters, USA) and Ezinfo 2.0 software (Waters, USA) and dealt with the multi-analysis including the principal component analysis (PCA) and partial least squares-discriminate analysis (PLS-DA), and we acquired the HepG2 cell metabolic profile (in Fig. 1). In Fig. 1a, the PCA score plot, we can find that there is clearly clustering and distinguishing between the treated groups (circle 2, 3, and 4) and the control groups (circle1); in Fig. 1b, the 3D PLSD-A score plot, the separation trending was further magnified. So, it demonstrated that the graphene could disturb the metabolic pathway of HepG2 cells (even low dose $25 \mu \mathrm{g} \cdot \mathrm{mL}^{-1}$ ), from the low dose to the high dose, the metabolism trajectory of HepG2 cell displayed the clockwise change and the pronounced dose-dependent. Combined the loading plot (Fig. 1c) with VIP score plot (Fig. 1d), we acquired 336 potential biomarker and threshold value setting VIP $>1$.

The potential biomarkers were suffered from the the MS/MS analysis by the precursor ion scan and retention time, then the mass data in integrated with the free database METLIN, metabolite, ChemSpider, HMDB, MassBank and KEGG to identify the structure of the potential markers (table1). The 12 metabolites are most of the nitrogen-containing compound including amino acid and its derivative, small molecule peptide, and so on 


\section{Univariate analysis}

Table 1 the identified markers

\begin{tabular}{llllll}
\hline Code & Rt & {$[\mathrm{M}+\mathrm{R}]$} & Data & Formula & Name \\
\hline M1 & 3.15 & {$[\mathrm{M}+\mathrm{H}]+$} & 307.323 & C10H17N3O6S & Glutathione \\
M2 & 2.78 & {$[\mathrm{M}+\mathrm{H}]+$} & 252.2267 & C10H12N4O4 & Deoxyinosine \\
M3 & 6.75 & {$[\mathrm{M}+\mathrm{H}]+$} & 488.324 & C14H26N4O11P2 & Citicoline \\
M4 & 0.56 & {$[\mathrm{M}+\mathrm{H}]+$} & 115.1305 & C5H9NO2 & Proline \\
M5 & 0.97 & {$[\mathrm{M}+\mathrm{H}]+$} & 145.2459 & C7H19N3 & Spermidine \\
M6 & 1.60 & {$[\mathrm{M}+\mathrm{Li}]+$} & 184.0431 & C6H11NOS2 & Sulforaphane \\
M7 & 0.53 & {$[\mathrm{M}+\mathrm{H}]+$} & 221.0320 & C6H9N2O5P & 3-(Imidazol-4-yl)-2-oxopropyl \\
M8 & 3.62 & {$[\mathrm{M}+\mathrm{H}]+$} & 184.0634 & C5H13NO4S & Unkown \\
M9 & 4.19 & {$[\mathrm{M}+\mathrm{H}]+$} & 226.1192 & C10H15N3O3 & 1-(Methylnitrosoamino)-4-(3-pyridinyl)-1,4-but \\
& & & & & anediol \\
M10 & 2.13 & {$[\mathrm{M}+\mathrm{Na}]+$} & 275.0689 & C16H12O3 & 1-[2-(4-Hydroxyphenyl)-1-benzofuran-3-yl]eth \\
& & & & & anone \\
M11 & 8.39 & {$[\mathrm{M}+\mathrm{ACN}+\mathrm{H}]$} & 804.4819 & C44H42N8O5 & Trp Trp Trp Trp \\
M12 & 7.96 & {$[\mathrm{M}+\mathrm{H}]+$} & 466.1759 & C20H27N5O6S & Unkown \\
\hline
\end{tabular}

The signal intensity of potentials biomarkers were dealt by the univariate analysis (analysis of variance). All the change trending of metabolites were displayed by the box-figure (Fig.2), we can find that signal intensity of five metabolites (M2, M6, M7, M10, M11) are decreasing with the graphene concentration, that is the signal intensity of metabolite is inversely proportional to the added graphene dose, in contrast, that of the others ( 8 metabolites) are increasing with the graphene concentration. It demonstrated that the markers could were intensively disturbed by the graphene. The significant differences values were acquired by virtue of all the treated groups compared with the control group.

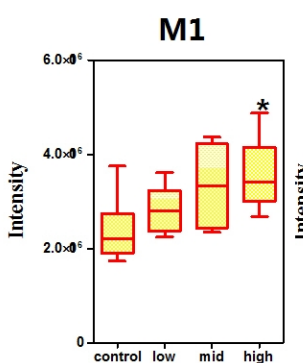

M7

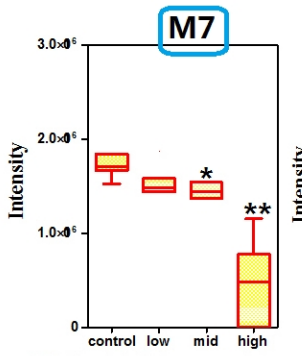

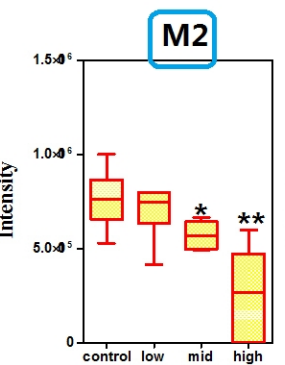

M8

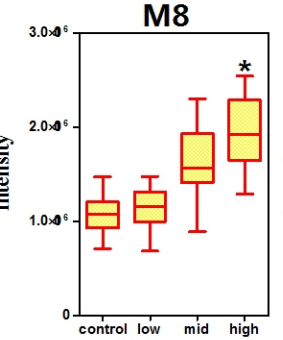

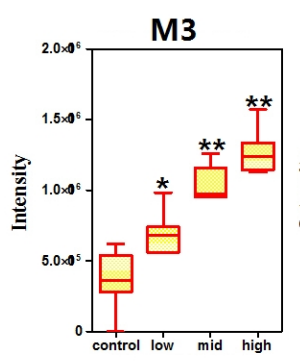

M9

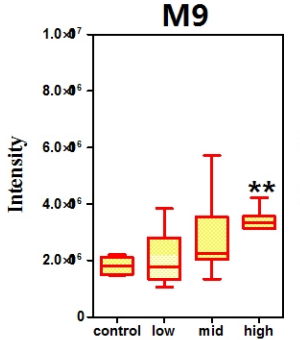

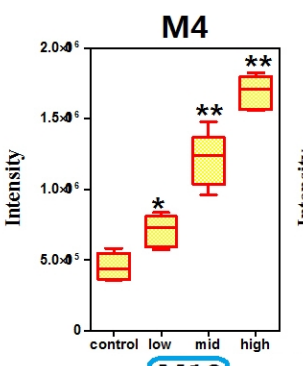

M10

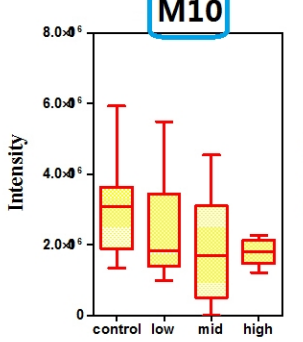

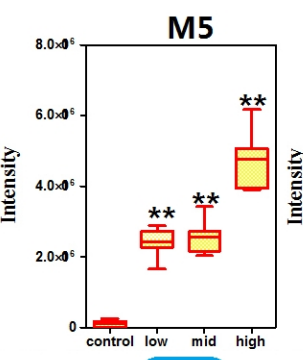

M11

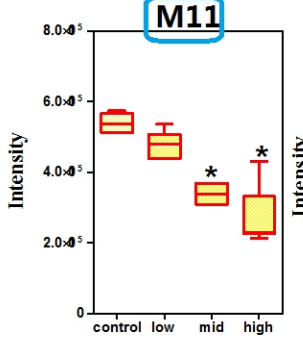

M6

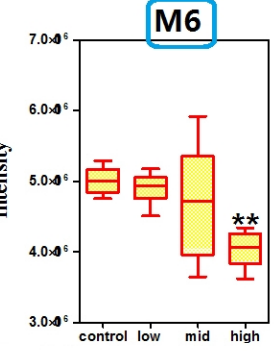

M12

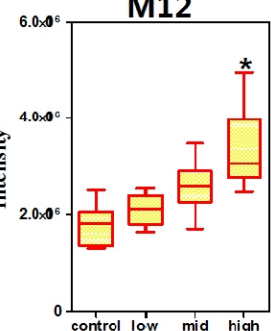

Fig.2 The Box-Figure univariate analysis. Statistical significance were displayed by compared the treated group (Low, Middle, and High dose group) with the Control group. Significant difference stands as “*” $p<0.05$, and “**” $p<0.01$.

\section{Pathway analysis}

All the biomarkers were input the KEGG, HMDB, MetPA, and SMPD database, we obtained the the disturbed metabolism pathway of HepG2 treated with graphene[3]. According to the pathway impact value (setting threshold value $>0.1$ ), we choose the disturbed metabolism pathway glutathione 
metabolism and arginine and proline metabolism (Fig. 3). The two disturbed metabolism pathway were disturbed by glutathione (M1), proline(M4), and spermidine(M5). In Fig. 2, we can find that the changed trending of these 3 markers, the glutathione, proline, and spermidine are also displyed increasing with the graphene dose.

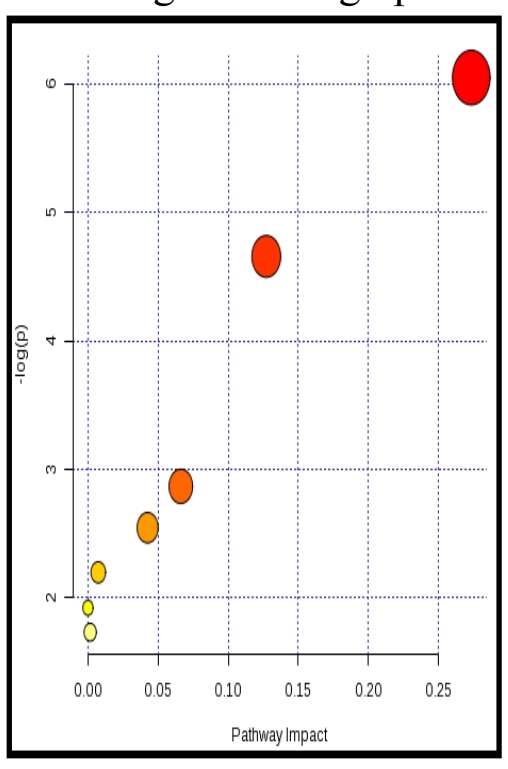

Fig. 3 pathway impact

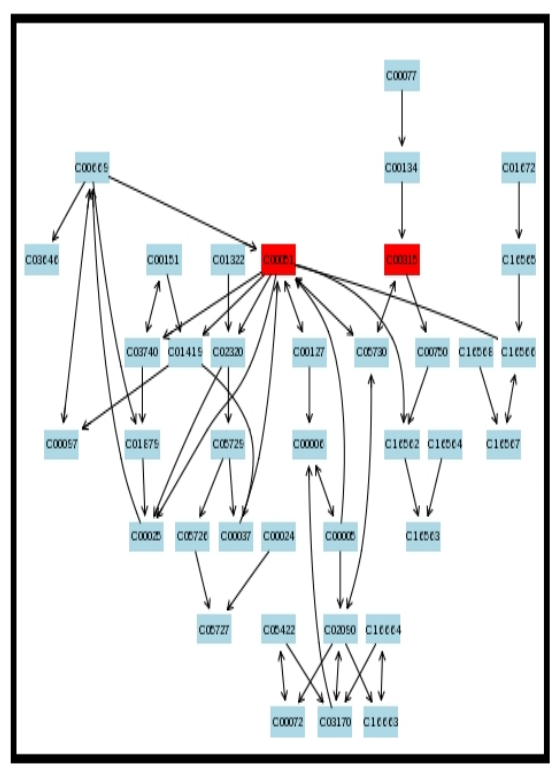

Fig. 4 Glutathione metabolism

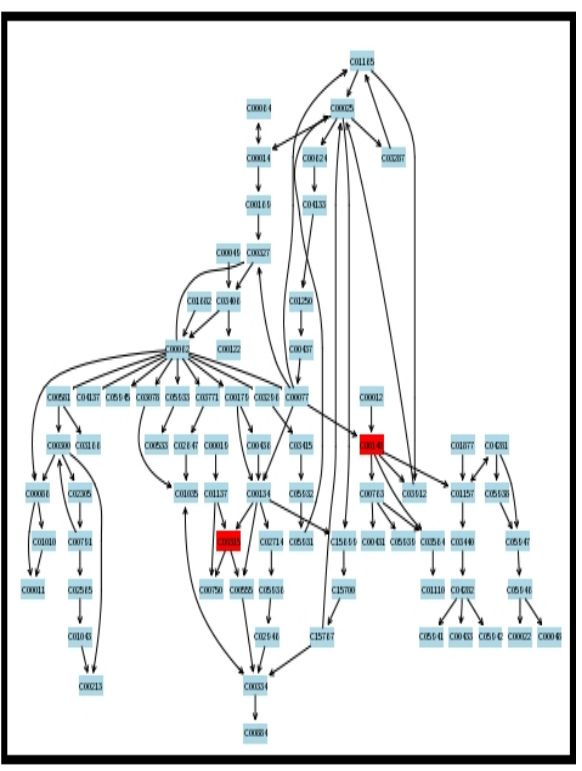

Fig . 5 arginine and proline metabolism

The two pathways of glutathione metabolism (Fig. 4) and arginine and proline metabolism (Fig. 5) play an important role in cell living activities. [4] Glutathione is mainly used to deal with the toxic waste and help detoxify, the crucial thiol (-SH) group play an important role in antioxidant. In liver, it can be used to conjugate reaction with the toxic chemicals and dispose them for detoxify. Additionally, the cell can help construct the red and white cell and improve the immunity. It can also be used to clinical to reduce the damages of the chemotherapy and radiation in cancer treatments, and can reduce the toxicity of heavy metal. Glutathione can also help the conjugation reactions and reduction reactions in in cytosol, microsomes, and mitochondria, and so on. The proline can be used forming the protein, moreover, it plays an important role in constructing the collagen. The pathway of arginine and proline metabolism is involved in the urea cycle. Spermidine is thought to improve the stabilize of some membranes and nucleic acid structures. In Fig. 3, Fig.4, and Fig.5, we can find that the homeostasis of the mentioned pathways was disturbed, so the HepG2 biological balance may be perturb by graphene. We should focus on this perturbation before widely usage this kind of nanomaterials. The further mechanism is still needed to be done.

\section{Summary}

In this study, we can find that the metabolites of HepG2 cells were disturbed by graphene, and two metabolism pathways including the glutathione metabolism and arginine and proline metabolism were disturbed by graphene. So, in order to make good use of the graphene nanomaterials, we should focus on these possibility perturbations of cells.

\section{References}

[1] G.X, M. N. Assessment of the toxic potential of graphene family nanomaterials. Journal of food and drug analysis, 22 (2014) 105-115.

[2] J. GZ, Li X, Zh. N, Q. J.Q, X. H.Y., L. SM. Metabolomics study on the cytotoxicity of graphene. Rsc Adv, 4(2014) 44712-7.

[3] X.J, S.I.V, Han B, Wishart DS. MetaboAnalyst 3.0-making metabolomics more meaningful. Nucleic acids research43 (2015) 251-257. 
[4] W. DS, F. A, K. C, et al. SMPDB: The Small Molecule Pathway Database. Nucleic Acids Res. 38(2010) (Database issue) 480-487. 